\title{
Lafora body disease presenting as neuropsychiatric illness
}

\author{
Bhat $\mathbf{M}^{1}$, Joshi $\mathbf{S}^{2}$
}

${ }^{1}$ Dr Minakshi Bhat, Lecturer, Pediatrics, ${ }^{2}$ Dr Surekha Joshi, Professor and Head, Pediatrics; both authors are affiliated with Terna Medical College, Nerul, Navi Mumbai, Maharashtra, India.

Address for Correspondence: Dr Minakshi Bhat, Lecturer Department of Paediatrics, Terna Medical College, Nerul, Navi Mumbai. Email ID: minakshi_libra@yahoo.in

\begin{abstract}
Background: Lafora disease is a rare progressive myoclonus epilepsy with an autosomal recessive inheritance characterized by seizures, myoclonus and progressive cognitive decline. Case characteristics: An 11 year old boy presented with complaints of aggressive behavior, abnormal movements and declining intellectual function and was earlier treated as a case of attention deficit hyperactivity disorder. The child was subjected to Axillary skin biopsy as a part of evaluation and diagnosed as a case of Lafora body disease. parental counselling done and the child was discharged on symptomatic treatment. Message: Lafora body disease should be kept in mind while evaluating a child with neuropsychiatric manifestations.
\end{abstract}

Key words: Lafora body, Myoclonus, Aggressive behaviour

\section{Introduction}

Lafora body disease (LBD) is a rare neurometabolic disorder of autosomal recessive inheritance, which is generally caused by a mutation in EPM2A or EPM2B genes $[1,2,3,4]$. The onset usually occurs in childhood or adolescence. It was first described as a progressive myoclonus epilepsy by Lafora and Gluech in 1911. It is a rare disease characterized by epilepsy, myoclonus, dementia and the presence of Lafora bodies in various tissues $[5,6]$. A number of mental health manifestations have been reported including depression, psychosis, and personality changes. Dementia is common with the disorder, as well as intellectual disability. In this report we present a case who presented with predominant features of aggression, impulsivity and abnormal behaviour.

\section{Case Report}

An 11 year old male child was admitted to our hospital with complaints of hyperactivity, impulsiveness, abnormal movements and declining intellectual function. His detailed history revealed that he was born of non consanguineous marriage, first in birth order with uneventful perinatal period. The patient had attained all milestones as per age till four years and was apparently alright except two episodes of febrile convulsions between 2 to 3 year of age. He was noticed to have difficulty in writing and began to show signs of hyperactivity, impulsive behavior and declining school performance from 6 year onwards. On inquiry it was learnt that child had to be physically restrained as he would be found to be absconding from home and wondering around if left unattended. Stanford Binet intelligence test performed on child at 7 years of age showed IQ 72 and SQ 84. After consultations with pediatric neurologists and psychiatrists, the child was labelled as a case of attention deficit hyperactivity disorder (ADHD) and started on atomexitin.

He began to have multiple episodes of generalized tonic clonic seizures and was noticed to have repetitive movements of hands since 8 years of age and was receiving several anticonvulsants with little improvement. He began to show progressive cognitive and mental decline from 10 year onwards. At our center, on examination the patient was conscious but often disoriented, uncooperative and had ataxia, dysarthria, hypotonia, and hyporeflexia, In addition he was noticed

Manuscript received: $14^{\text {th }}$ August 2016

Reviewed: $26^{\text {th }}$ August 2016

Author Corrected; $7^{\text {th }}$ September 2016

Accepted for Publication: $19^{\text {th }}$ September 2016 
to have myoclonus of hands. However the child was uncooperative for detailed visual assessment. He was investigated for wilson's disease, subacute sclerosing panencephalitis (SSPE) and other neurodegenerative disorders associated with myolonic seizures. His laboratory investigations showed normal blood count, renal function and liver function tests. Serum ceruloplasmin was normal and urinary coppor was mildly elevated but d-pencillamine challenge test was negative. Measles antibody IgG was negative. Magnetic resonance imaging (MRI) of brain done outside a year ago, was normal. Electroencephalography (EEG) evaluation showed diffuse and non localized multiple spikes and slow wave forms. As a part of evaluation for myoclonic seizures he was subjected to biopsy of axillary sweat gland duct cells which showed PAS stain pink staining oval/globoid bodies, characteristic of LBD. His genetic study and repeat MRI could not be done due to financial constraints.

\section{Discussion}

LBD is a form of progressive myoclonus epilepsy beginning from age 5 to 20 years characterized by generalized tonic-clonic (GTC) seizures, visual hallucinations (occipital seizures), resting and action (fragmentary) myoclonus, Progressive neurological deterioration including ataxia, cognitive deterioration [7]. Though Seizures, myoclonus or learning disability may be the first symptom in majority of the patients [8, 9 , 10, 1]. But our case was an exception as hyperactivity, aggressive and abnormal behavior associated with learning disability were the earliest manifestations which mislead treating neurologists and psychiatrists.

There is only one case mentioned in literature where a case of LBD presented with disruptive and distractive behavior [12]. Rapid progressive dementia and global cognitive dysfunction often develop 2-6 years after the disease onset though convulsions may initially respond well to antiepileptics. Visual deterioration with normal fundus is common. Death occurs at a mean of 2-10 years after the diagnosis [13.14]. Our case also showed deterioration in cognitive function four years after disease onset. Repeat MRI brain could not be done to document atrophy and other changes; though initial MRI brain was normal. Literature also mentions that the cranial images may be normal intially, but with the progression of the disease, diffuse atrophy may be seen [13.14]. The diagnosis may be confirmed by the demonstration of typical spherical PAS-positive inclusion bodies in the brain, spinal cord, heart, liver, skeletal muscle, and axillary sweat gland duct cells [15]. In our patient the diagnosis of LBD was confirmed by demonstration of typical lafora bodies from axillary skin biopsy.

\section{Conclusion}

Instead of usual presentation LBD can rarely present with predominant neuropsychatric manifestations. Hence LBD should be kept in mind while evaluating such a case.
Aknowledgement- We sincerely thank Dr Udhay Khopkar (consultant dermatologist) and Dr. Parag Sharma (associate professor in department of dermatology) for doing skin biopsy.

Funding: Nil, Conflict of interest: Nil

Permission from IRB: Yes

\section{References}

1. Baykan B, Striano P, Gianotti S et al . Late-onset and slow-progressing Lafora disease in four siblings w i t h EP M2B mutation. Epileps ia 2005;(46):1695-1697.

2. Erbay H, Balci C, Tomatir E etal. Refractory status epilepticus in intensive care unit: A case of Lafora body disease. Inter J Emer Inten Care Med 2003; E6 (2):[online serial].

3. Minassian BA, Ianzano L, Meloche M et al. Mutation spectrum and predicted function of laforin in Lafora's progressive myoclonus epilepsy. Neurology 2000; (55):341-6.

4. Wallace R. A second gene for Lafora disease. Epilepsy Curr 2004;(4):82-83.

5. Kaplan KJ, Nelson BL. Pathologic quiz case myoclonic epilepsy and cognitive decline in a 19-year old man. Arch Pathol Lab Med 2003;(127): 231-2.

6. Ünver O, Demirkesen C, Uysal S. Lafora disease: a progressive myoclonic epilepsy. Turk Arch Ped 2011; (46): 161-3. R(5)

7. Satishchandra $P$ and Sinha S. Lafora body disease: NIMHANS experience. CURRENT SC IENCE 2013; $105: 6$

8. Berge A. Minassian BA. Lafora's Disease: Towards a Clinical, Pathologic, and Molecular Synthesis. PEDIAT NEURO2001; 25 R(7) 
9. Acharya, J. N., Satishchandra, P, Asha, T etal. LBD in south India - a clinical, electrophysiological and pathologicalstudy. Epilepsia, 1993; (34) 476-487.

10. Sinha, S, Satishchandra, P, Yasha, T etal. Progressive myoclonic epilepsy: a clinical, electrophysiological and pathological study from south India. J. Neurol. Sci.,2007, (252), 16-23.

1I. Rapin, I., Myoclonus in neuronal storage and Lafora diseases. In Myoclonus - Advances in Neurology (eds Fahn, S., Marsden, C. D and Von Woert, M. H.), Raven Press, New York, 1986, vol. 43,pp. 65-85.
12. Palmer GA, Mark FH. Medical and Mental Health Complications of Lafora Disease: A Case Report. Ment Health Asp of Dev Disability January/ February/ March 2007; 10 R(I)

13. Zupanc ML, Legros B. Progressive myoclonic epilepsy. Cerebellum 2004; 3: 156-171

14. Bektaş O, Yılmaz A, Okcu AH etal. A type of progressive myoclonic epilepsy, Lafora disease: A case report. EastJour of Med 2013; (18):34-36 R(2)

15. Comoglu S, Ozbakir S,Pesinci E. Diagnosis of Lafora Body Disease by Axillary Skin Biopsy.A case report. Turk J Med Sci 2006; (36) 235-237 R(3)

\section{How to cite this article?}

Bhat M, Joshi S. Lafora body disease presenting as neuropsychiatric illness. Int. J Pediatr Res. 2016;3(9):712714.doi:10.17511/ijpr.2016.i09.15. 